\title{
Case Report: Cathodal Transcranial Direct Current Stimulation to Ameliorate the Frequency and Severity of Motor Tics: A Case Study of Tourette Syndrome
}

\author{
Sanaz Tajadini ${ }^{1}$ (D), Neda Mohammadi ${ }^{1,2}$ (D), Mahshid Tahamtan ${ }^{1,2}$ (i), Mohammad Nami ${ }^{1,2,3,4^{*}}$ (i) \\ 1. DANA Brain Health Institute, Shiraz, Iran. \\ 2. Department of Neuroscience, School of Advanced Medical Sciences and Technologies, Shiraz University of Medical Sciences, Shiraz, Iran \\ 3. Clinical Neurology Research Center, Shiraz University of Medical Sciences, Shiraz, Iran. \\ 4. Academy of Health, Senses Cultural Foundation, Sacramento, USA.
}

\begin{tabular}{l|l}
$\begin{array}{c}\text { Use your device to scan } \\
\text { and read the article online }\end{array}$ & $\begin{array}{l}\text { Citation Tajadini S, Mohammadi N, Tahamtan M, Nami M. Cathodal Transcranial Direct Current Stimulation to Amelio- } \\
\text { rate the Frequency and Severity of Motor Tics: A Case Study of Tourette Syndrome. JAMSAT. 2018; 4(1):21-26. https://doi. } \\
\text { org/10.32598/jamsat.4.1.21 }\end{array}$ \\
doi $\mathbf{h t t p s : / / d o i . o r g / 1 0 . 3 2 5 9 8 / j a m s a t . 4 . 1 . 2 1 ~}$
\end{tabular}

c) (i) (5)

Article info:

Received: 10 Oct 2017

Accepted: 25 Jan 2018

Available Online: 01 Mar 2018

Keywords:

Transcranial Direct Current

Stimulation, Tourette

Syndrome, Tic disorder,

Quantitative EEG

\section{ABSTRACT}

Objectives: Dysregulated neuronal activity in Cortico-Striato-Thalamo-Cortical (CSTC) networks is primarily described as the pathophysiology of Tourette Syndrome (TS). New neuromodulation techniques including noninvasive transcranial Electrical Stimulation (tES) are increasingly considered among potential therapeutic options for TS.

Materials and Methods: To test the therapy protocol with transcranial Direct Current Stimulation (tDCS) a 31 year-old male patient with TS was selected to be administered for 10 sessions over five days of bilateral cathodal tDCS ( 30 minutes, $2 \mathrm{~mA}$ ) on $\mathrm{C}_{3}$ and $\mathrm{FC}_{5}$, while the anode electrodes were placed on $\mathrm{FP}_{2}$.

Results: Tic severity and frequency were subjectively reported by the subject following the treatment on weekly basis and treatment response was objectively assessed by two experts. The patient noted that he was able to control his unwanted movement after over one year follow-up.

Discussion: The current study results together with earlier reports support the therapeutic effects of cathodal tDCS on tics in patients with TS. Randomized sham-controlled studies are required to shed more light on such therapeutic impact and the optimized protocols. 


\section{Highlights}

- Ten sessions of tDCS can ameliorate some problems in Tourette syndrome.

- The number of motor and vocal tics decreased following tDCS treatment.

- Unwanted movement was still controllable after one year follow-up.

\section{Plain Language Summary}

Tourette Syndrome (TS) is defined as a childhood neurodevelopmental condition which has harmful impact on social functioning. People with TS may show various motor and vocal tics. Although medications are available for tics, patients prefer to use non-drug and safer strategies to treat or manage their tic symptoms. In this regard, transcranial Direct Current Stimulation (tDCS) may be considered safe and efficient in ameliorating tic severity.

To test the therapy protocol with tDCS, a 31-year-old man with TS was selected to receive 10 sessions (over five days) of bilateral cathodal tDCS. In a three-month period of follow-up, the number of motor and vocal tics dramatically decreased. In sum, the current study results support the use of tDCS in patients with TS who are reluctant to use traditional treatment methods or concerned about the toxic effects of the medicines.

\section{Introduction}

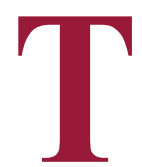

ourette Syndrome (TS) is defined as a childhood neurodevelopmental condition associated with dysregulation in Cortico-Basal Ganglia-Thalamo-Cortical (CBGTC) loops [1]. It is estimated that TS affects $0.77 \%$ of the population presented through various motor and vocal tics $[2,3]$. People with DIC disorders including TS may show tics such as raising the eyebrows, turning the eyes, shaking their head and repeating or uttering words and phrases [4]. Fluctuation in appearance, severity and durability of sudden or repetitive tics can be observed in different people with the same syndrome [5]. Besides biological origins, environmental stress and internal psychological factors play a defining role in the course of tic disorder $[6,7]$. On the other hand, comorbid disorders including Obsessive-Compulsive Disorder (OCD), learning disabilities, Attention Deficit Disorder (ADD)/ Attention Deficit Hyperactivity Disorder (ADHD), anxiety, and mood disorder are proposed as contributing factors to tic disorders $[8,9]$.

To date, the medical approach to tics management includes pharmacotherapy as the first-line intervention, which might be found ineffective or intolerable in some if not many patients $[10,11]$. Therefore, as with most chronic TS, patients tend to shift towards non-drug and safer strategies to treat or manage their tic symptoms. In this regard, transcranial Direct Current Stimulation (tDCS) as an alternative and noninvasive intervention in clinical applications may be considered safe and efficient in ameliorating tic symptoms. Meanwhile, evidence to support the role of tDCS to remediate TS is still insufficient [12]. The current study aimed at reporting a patient with medication refractory TS that demonstrated sustainable clinical response to a course of tDCS.

\section{Materials and Methods}

\section{Case description}

A 31-year old male presented with a long standing history of tic disorder. His first tics were observed in his early childhood. Later, his symptoms gradually aggravated and started to dramatically impact his quality of life. Throughout the course of his symptoms, tics predominantly involved his face, shoulders and stomach. He had uncontrolled repetitive movements in his face and hands, shrugging his shoulder and repeatedly uttering the same words while speaking. Over the past several years, the patient took Pimozide $20 \mathrm{mg}$ qd and Haloperidol $5 \mathrm{mg}$ qd, with minimsl or suboptimal response as well as notable untoward effects including extrapyramidal symptoms, hypersomnolence, and dizziness.

\section{Baseline evaluation}

Having signed the written consent, the patient underwent a 19-channel (10-20 system) Mitsar monopolar setup Quantitative Electroencephalographic (QEEG) study upon resting, eyes-open state whilst his tic events were concurrently marked on EEG tracing using the stim-tracker setup. After preprocessing the data to obtain denoised EEG using the Neuroguide software (Applied Neuroscience, Inc. Largo, FL; version 2.8.9.2016; USA), event- 
Absolute power (uV sq)

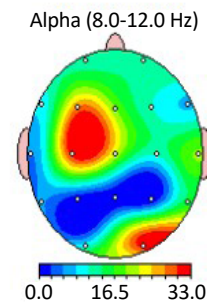

Relative power (\%)

Amplitude asymmetry
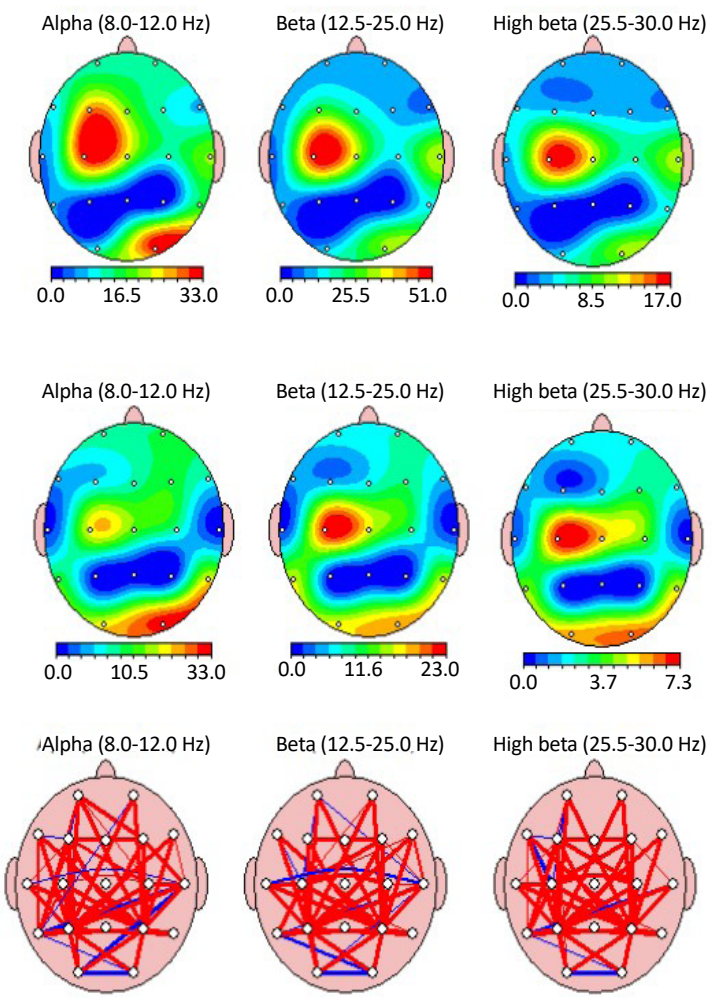

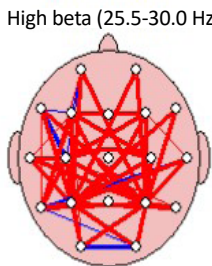

Figure 1. The color-coded quantitative EEG brain map upon resting, eyes-open state

associated segments (three seconds) were extracted from the tracing and separately analyzed.

The analysis revealed an increased alpha $(8-12 \mathrm{~Hz})$ Current Spectral Density (CSD) in the left frontocentral area $\left(\mathrm{C}_{3}\right)$. Moreover, in the FFT relative power analysis, an increased relative beta $(13-30 \mathrm{~Hz})$ power was noted in bioccipital and left central brain regions. Meanwhile, there was an amplitude asymmetry favoring frontal brain regions for alpha and beta frequency bands. Additionally, Z-score FFT coherence analysis demonstrated hyper-coherence in the left inferior-frontal brain $\left(\mathrm{FC}_{5}\right)$ and central brain (FCZ) regions (Figure 1).

\section{Experimental protocol}

\section{The tDCS procedure}

The entire procedure was performed in an academic-based Neuroscience Laboratory adhering to the ethical and professional standards laid down by the Department of Neuroscience, SUMS, for applied neuroscience service provision. The patient underwent a course of dual-channel cathodal tDCS, twice a day for five consecutive days using the Neuro-Stim two-channel tES device (Medina-Teb, Tehran).
The protocol involved a 20-second ramp-up period followed by duration of 30 minutes of stimulation at $2 \mathrm{~mA}$ in a fixed schedule. The anodal sites were placed above the right frontopolar area $\left(\mathrm{FP}_{2}\right)$, while the cathodal electrodes were positioned over the left motor cortical $\left(\mathrm{C}_{3}\right)$ and left inferior frontal $\left(\mathrm{FC}_{5}\right)$ brain regions (Figure 2). Cathodal electrodes were placed bilaterally over pre-motor cortical areas $\left(\mathrm{C}_{3}\right.$, $\mathrm{FC}_{5}$ ). Anodal electrodes were placed over right frontal area $\left(\mathrm{FP}_{2}\right)$. A 2-mA current was applied for 30 minutes.

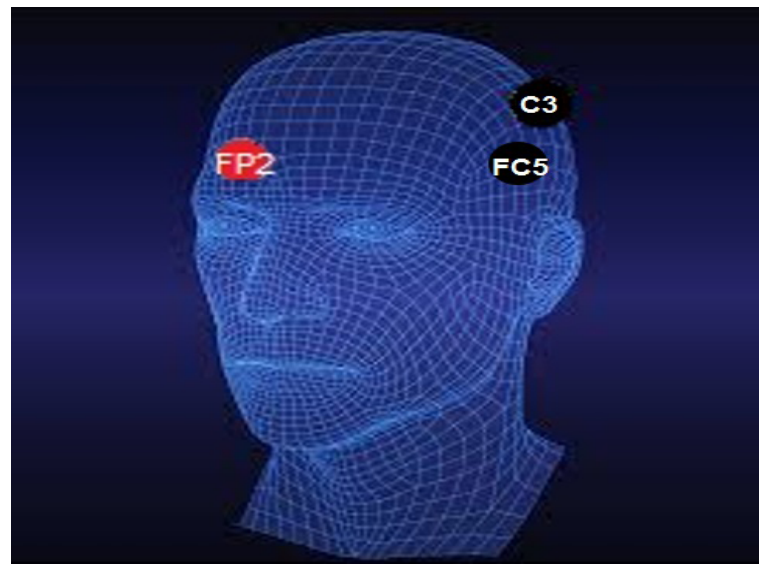

JAMSAT

Figure 2. Dual-channel cathodal tDCS intervention 


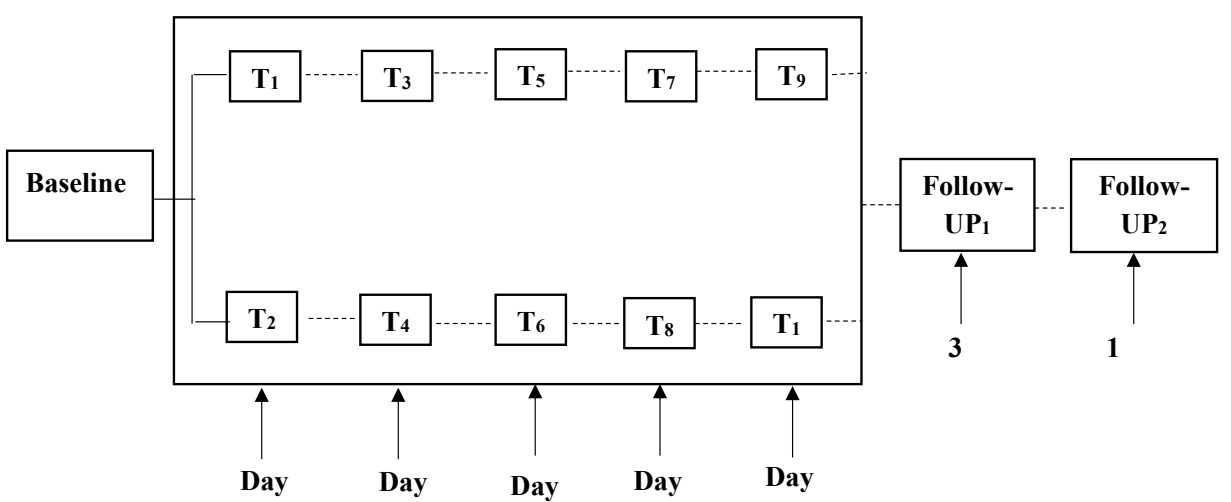

Figure 3. Time-line of the 5-day stimulation for twice daily sessions and clinical response follow-up

Table 1. Stimulation parameters and results of studies investigating the effect of $\mathrm{tDCS}$ in TS

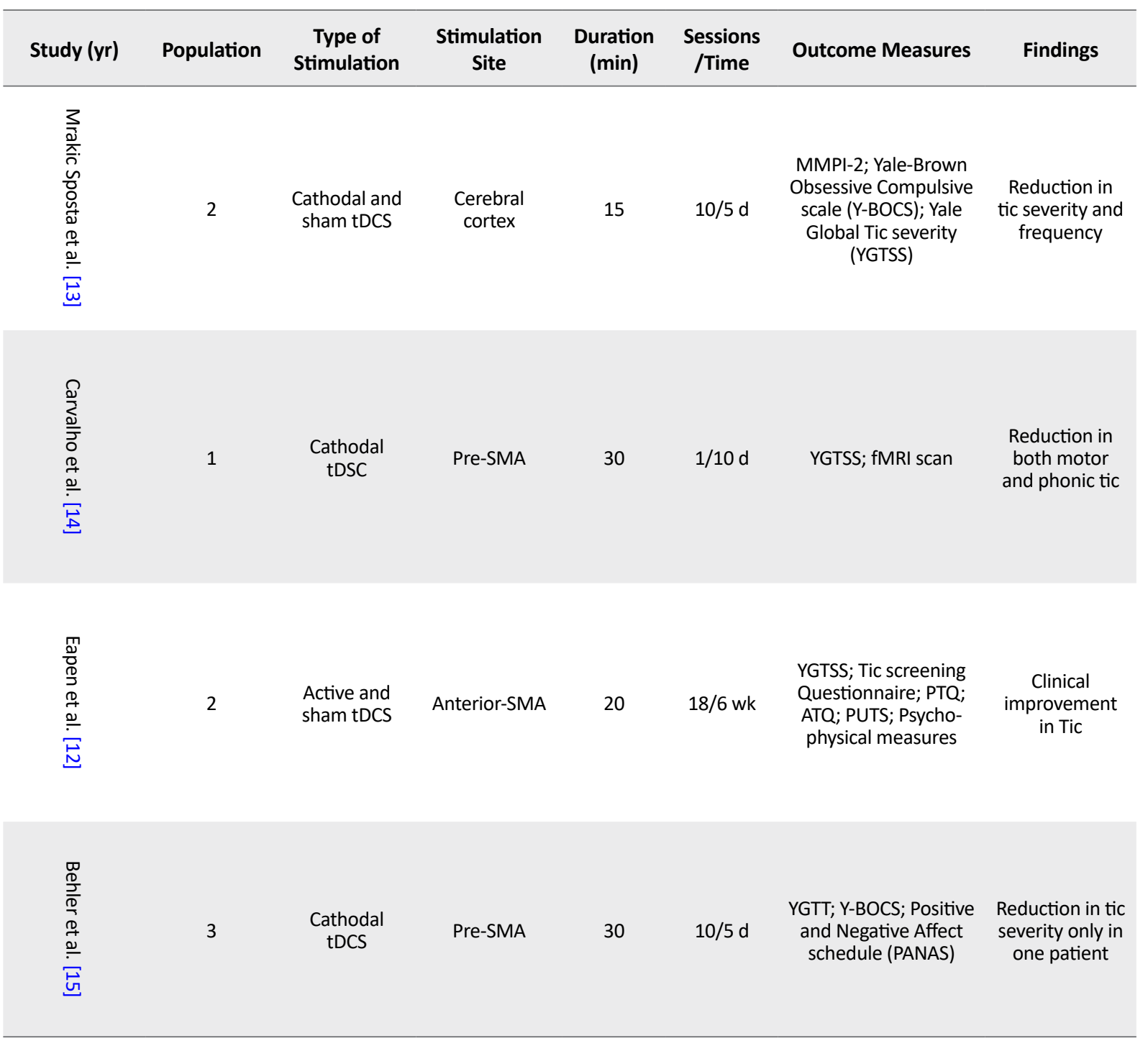




\section{Results}

The patient completed five days of stimulation sessions on twice daily basis (e.g. 9:00 and 20:00). In a threemonth period of follow-up visit after 10 sessions of tDCS application, the number of motor and vocal tics dramatically decreased. The condition was both reported by the subject (through weekly visual analogue scale records) and approved by a neurologist and a clinical neuroscientist. The patient noted that he was able to control his unwanted movement after one year follow-up; while, after a year, a weak stomach tic was reported. He felt like he was being tickled from the inside while it was not reported disabling or even unpleasant. The timeline of the remediation schedules is displayed in Figure 3.

Classically described as a chronic neuropsychiatric disorder, TS is further defined by its harmful impact on social functioning and self-image [5]. Although therapeutic pharmacological resources for tics are accessible, the information about the differential impact of cathodal and anodal tDCS intervention is scarce in the literature.

In the current study patient, a consecutive 10-session course of cathodal suppression transcranial Direct Current Stimulation (tDCS) over motor cortical and Broca's areas diminished tic severity and the symptoms disappeared after one year since the intervention. This result followed a report in 2008 in which two adult cases with TS that received cathodal tDCS over the left motor areas of the cerebral cortex revealed significant and sustainable symptom control [13]. According to this former study, the entire spectrum of tic symptoms in both patients was gradually improved by tDCS therapeutic intervention. Likewise, another case report on a 16 year-old male with vocal and motor tics, demonstrated that cathodal tDCS over the pre-Supplementary Motor Area (SMA) was associated with a significant reduction in the percentage of tic severity compared to that of the baseline [14].

In a review and preliminary study [12], others showed that tDCS application was an important factor in management of TS and reduction of impairment severity. In other words, some patients with tic disorder had a more rapid decline in frequency and intensity of tics over the course of treatment. Furthermore, the latter results were partly confirmed in a recently published case series which investigated the therapeutic effects of cathodal tDCS to SMA areas in three patients with tic disorder [15]. The current study results indicated that significant improvement was achieved in one out of three patients. Meanwhile, no changes were observed during cathodal stimulation in two patients. The results indicated that tDCS intervention was not sufficiently effective in ameliorating tic symptoms. The details related to tDCS efficacy in tic disorders are outlined in Table 1.

Due to the unclear efficiency of tDCS to reduce tic symptoms, it is yet to be proven if specific stimulation parameters are taken into account. Therefore, electrode placement, electrode size, current dosage, and frequency of remediation may constitute prominent predictors for efficacy of tDCS in TS [15]. Although the reduction in tic frequency and severity were observed in the current study patient's symptoms without adverse effects, the outcome of this single case report is behind comprehensive. However, considering the long term follow-up visit, effects of pharmacotherapy, and differences in age at onset of disorder, differences in pathology and etiology may play an important role in influencing the outcomes during neuro-stimulation. Moreover, the current study resultS can motivate further studies to focus on the mentioned indicators to evaluate $\mathrm{tDCS}$ as a viable therapy to facilitate the functional recovery and reduce TS manifestations.

\section{Ethical Considerations}

\section{Compliance with ethical guidelines}

The informed consent was obtained from the subject declaring that his clinical data would be used in the institutional research databases of the current study.

\section{Funding}

This research did not receive any specific grant from funding agencies in the public, commercial, or not-forprofit sectors.

\section{Authors contributions}

Writing the manuscript and data analysis: Sanaz Tajaddini; Data acquisition and project administration: Neda Mohammadi and Mahshid Tahamtan; Supervision and designing treatment protocol: Mohammad Nami.

\section{Conflict of interest}

The authors declared no conflict of interest.

\section{Acknowledgements}

The authors appreciate the Neuroscience Laboratory of Shiraz University of Medical Sciences to provide the instruments for performing this project. 


\section{References}

[1] Albin RL, Mink JW. Recent advances in Tourette Syndrome research. Trends in Neurosciences. 2006; 29(3):175-82. [DOI:10.1016/j.tins.2006.01.001] [PMID]

[2] Association AP. Diagnostic and Statistical Manual of mental disorders (DSM-5®). Philadelphia: American Psychiatric Association; 2013. [DOI:10.1176/appi.books.9780890425596]

[3] Knight T, Steeves T, Day L, Lowerison M, Jette N, Pringsheim T. Prevalence of tic disorders: A systematic review and meta-analysis. Pediatric Neurology. 2012; 47(2):7790. [DOI:10.1016/j.pediatrneurol.2012.05.002] [PMID]

[4] Kompoliti K, Verhagen L. Encyclopedia of movement disorders. Cambridge: Academic Press; 2010. [PMID]

[5] Perera H, Abayanayaka C. Management of severe pediatric Tourette Syndrome resistant to drug treatment. Case Reports in Psychiatry. 2012; 2012:497160. [DOI:10.1155/2012/497160]

[6] Lin H, Katsovich L, Ghebremichael M, Findley DB, Grantz $\mathrm{H}$, Lombroso PJ, et al. Psychosocial stress predicts future symptom severities in children and adolescents with Tourette Syndrome and/or obsessive-compulsive disorder. Journal of Child Psychology and Psychiatry. 2007; 48(2):157-66. [DOI:10.1111/j.1469-7610.2006.01687.x] [PMID] [PMCID]

[7] Leckman JF, Cohen DJ. Tourette's syndrome--tics, obsessions, compulsions: Developmental psychopathology and clinical care. Hoboken: John Wiley \& Sons; 1999.

[8] Simpson HA, Jung L, Murphy TK. Update on AttentionDeficit/Hyperactivity Disorder and tic disorders: A review of the current literature. Current Psychiatry Reports. 2011; 13(5):351-6. [DOI:10.1007/s11920-011-0223-1] [PMID]

[9] Eddy CM, Cavanna AE. Tourette Syndrome and obsessive compulsive disorder: Compulsivity along the continuum. Journal of Obsessive-Compulsive and Related Disorders. 2014; 3(4):363-71. [DOI:10.1016/j.jocrd.2014.04.003]

[10] Waldon K, Hill J, Termine C, Balottin U, Cavanna AE. Trials of pharmacological interventions for Tourette Syndrome: A systematic review. Behavioural Neurology. 2013; 26(4):26573. [DOI:10.1155/2013/626410] [PMID] [PMCID]

[11] Quezada J, Coffman KA. Current approaches and new developments in the pharmacological management of Tourette Syndrome. CNS Drugs. 2018; 32(1):33-45. [DOI:10.1007/ s40263-017-0486-0] [PMID] [PMCID]

[12] Eapen V, Baker R, Walter A, Raghupathy V, Wehrman JJ Sowman PF. The role of transcranial Direct Current Stimulation (tDCS) in Tourette Syndrome: A review and preliminary findings. Brain Sciences. 2017; 7(12):161. [DOI:10.3390/brainsci7120161] [PMID] [PMCID]

[13] Mrakic Sposta S, Marceglia S, Mameli F, Dilena R, Tadini L, Priori A. Transcranial direct current stimulation in two patients with Tourette Syndrome. Movement Disorders Journal. 2008; 23(15):2259-61. [DOI:10.1002/mds.22292] [PMID]

[14] Carvalho S, Gonçalves ÓF, Soares JM, Sampaio A, Macedo F, Fregni F, et al. Sustained effects of a neural-based intervention in a refractory case of Tourette Syndrome. Brain Stimulation: Basic, Translational, and Clinical Research in Neuromodulation. 2015; 8(3):657-9. [DOI:10.1016/j.brs.2014.12.008] [PMID]
[15] Behler N, Leitner B, Mezger E, Weidinger E, Musil RL, Blum B, et al. Cathodal tDCS over motor cortex does not improve Tourette Syndrome: Lessons learned from a case series. Frontiers in Behavioral Neuroscience. 2018; 12:194. [DOI:10.3389/fnbeh.2018.00194] [PMID] [PMCID] 